\title{
A Retrospective Survey among SARS-CoV-1 Infected Healthcare Workers after Three Years Post-Infection
}

\author{
Szu-Wei Huang ${ }^{1}{ }^{D}$, Aspiro Nayim Urbina ${ }^{2}$, Yi-Ming Arthur Chen ${ }^{3,4, *}$ and Sheng-Fan Wang $2,5,6, * \mathbb{D}$ \\ 1 Model Development Section, Basic Research Laboratory, Center for Cancer Research, \\ National Cancer Institute, Frederick, MD 21702, USA; szu-wei.huang@nih.gov \\ 2 Center for Tropical Medicine and Infectious Disease, Kaohsiung Medical University, \\ Kaohsiung 80708, Taiwan; aspiro.urbina@hotmail.com \\ 3 Graduate Institute of Biomedical and Pharmaceutical Science, Fu Jen Catholic University, \\ New Taipei City 242062, Taiwan \\ 4 Institute of Infectious Diseases and Vaccinology, National Health Research Institutes, \\ Miaoli County 35053, Taiwan \\ 5 Department of Medical Laboratory Science and Biotechnology, Kaohsiung Medical University, \\ Kaohsiung 80708, Taiwan \\ 6 Department of Medical Research, Kaohsiung Medical University Hospital, Kaohsiung Medical University, \\ Kaohsiung 80708, Taiwan \\ * Correspondence: 150110@mail.fju.edu.tw (Y.-M.A.C.); wasf1234@kmu.edu.tw (S.-F.W.); \\ Tel.: +886-2-2905-6251 (Y.-M.A.C.); +886-7-312-1101 (ext. 2350) (S.-F.W.)
}

check for updates

Citation: Huang, S.-W.; Urbina, A.N.; Chen, Y.-M.A.; Wang, S.-F. A Retrospective Survey among SARS-CoV-1 Infected Healthcare Workers after Three Years Post-Infection. Pathogens 2021, 10, 1078. https://doi.org/10.3390/ pathogens10091078

Academic Editors: Lawrence S. Young and Sonia Zuñiga

Received: 9 July 2021

Accepted: 23 August 2021

Published: 25 August 2021

Publisher's Note: MDPI stays neutral with regard to jurisdictional claims in published maps and institutional affiliations.

Copyright: (c) 2021 by the authors. Licensee MDPI, Basel, Switzerland. This article is an open access article distributed under the terms and conditions of the Creative Commons Attribution (CC BY) license (https:// creativecommons.org/licenses/by/ $4.0 /)$.
Abstract: Healthcare workers (HCWs) are on the frontline fighting several infectious diseases including SARS-CoV-1 and COVID-19. Coronavirus neutralizing antibodies (nAbs) were recently reported to last for a certain period. The factors affecting nAbs' existence remain unclear. Here, we retrospectively analyzed the factors correlating with nAbs' from SARS-CoV-1 long-term convalescence HCWs in Taiwan. One hundred and thirty SARS-CoV-1 convalescent patients were recruited between August 2006 and March 2007. Blood samples were collected to determine the anti-nucleocapsid (N) and anti-spike (S) antibodies' existence status and neutralization ability. Neutralization ability was measured using SARS-CoV-1 pseudotyped viruses. Statistical analysis of factors associated with anti-SARS-CoV-1 antibodies' existence status was determined using SAS software. $46.2 \%$ SARS$\mathrm{CoV}-1$ convalescent patients presented anti-N antibody after three years post-infection. Among sixty participants, ten participants co-presented anti-S antibodies. Eight participants with anti-S antibody displayed neutralization ability to SARS-CoV-1. The gender, age, and disease severity of participants did not affect the anti-N antibody existence status, whereas the anti-S antibody is significantly reduced in participants with old age $(>50$ years, $p=0.0434)$ after three years post SARS-CoV- 1 infection. This study suggests that age is an important factor correlated with the duration of SARS-CoV-1 protective antibody existence status.

Keywords: COVID-19; SARS-CoV-1; coronaviruses; convalescent; neutralizing antibodies

\section{Introduction}

Healthcare Workers (HCWs) have been on the frontline fighting several infectious diseases, including COVID-19, which may place them at high risk of becoming infected with various emerging or re-emerging diseases. Approximately 20 years ago, Taiwan had experienced a large-scale severe acute respiratory syndrome coronavirus 1 (SARS-CoV-1) outbreak, and many HCWs were infected, resulting in quarantine of HCWs and patients in hospitals, as well as several tertiary hospitals having shut-down [1]. Although many reports have studied SASR-CoV-1 pathogenesis and virus-induced protective neutralizing antibodies (nAbs), the prevalence and influence factors of long-term protective nAbs among SARS-CoV-1 HCWs remain not fully understood. 
The SARS-CoV-1 is responsible for the epidemic of severe acute respiratory syndrome (SARS) in 2003. During this time there was a series of outbreaks of SARS-CoV-1 nosocomial infections in Taiwan [1]. According to the World Health Organization, there were 364 SARS-CoV-1 confirmed cases by real-time RT-PCR and/or neutralizing antibodies (nAbs) test in Taiwan in 2003, and among them, 80\% were HCWs and patients living in the hospital. When a new pathogen infects humans, it is important to know whether or how long protective immunity can sustain and protect from re-infection. Some studies have reported that the anti-spike $(S)$ antibody in convalescent SARS-CoV-1 infected patients significantly waned after a few years post-infection [2,3]. Furthermore, it is still unclear regarding the deterioration rate of the specific antibodies against SARS-CoV-1, as well as the risk factors (such as age, sex or clinical characteristics) correlated with the anti-SARS antibodies existence status.

Recently, a novel coronavirus (SARS-CoV-2) emerged at the end of 2019 and caused the pandemic of coronavirus infectious disease 2019. The number and level of SARS-CoV-2 infected patients are significantly larger than the SARS-CoV-1. Therefore SARS-CoV-2 is posing a great threat to public health despite several COVID-19 vaccines being applied in different countries [4]. Currently, several data remain lacking, such as the possibility of secondary infection during a short period and how long the protective antibody may sustain. Understanding the duration of immune responses after coronavirus infection could provide valuable information for epidemiological assessments, therapies, and vaccines.

We previously collected the blood samples from SARS-CoV-1 confirmed HCWs after a three-year post-infection and studied the correlation of human-leukocyte antigen typing with SARS-CoV-1 infection [5]. Additionally, a recent report indicated that SARS-CoV-1 and SARS-CoV-2 shared several similarities [6]. The genome of SARS-CoV-2 has shown to be similar to SARS-CoV-1 (79\% similarity), with some proteins encoded in SARS-CoV-2 showing higher identity (more than 90\%) to SARS-CoV-1 [6]. The nAbs (primarily IgG) binding protein and the spike glycoprotein (S protein) share $77.5 \%$ identity between SARSCoV-2 and SARS-CoV-1 and share $74 \%$ identity of the receptor-binding domain. It is believed that the proteins with high identity between SARS-CoV-1 and SARS-CoV-2 have conserved the same mechanisms and functions. The nAbs from convalescent patients infected with SARS-CoV-1 have been reported to be able to cross-neutralize SARS-CoV-2 infection through a blockage of the viral entry step [7-9]. This implies that the SARSCoV-2 and SARS-CoV-1 could present the same protective epitope for producing nAbs. These similarities between SARS-CoV-1 and SARS-CoV-2 draw our interest to address the question regarding the duration of anti-SARS-CoV-1 protective antibodies' existence status and potential risk factors that may influence antibodies' expression capabilities. Therefore, we conducted a retrospective survey of nAbs' existence status using the samples from convalescent SARS-CoV-1 infected HCWs collected after three years post-infection.

\section{Materials and Methods}

\subsection{Sample Collection}

A total of 130 convalescent SARS-CoV-1 infected HCWs were recruited in this study after three years post-infection. The protocols for collecting and processing the blood samples from this cohort were described previously $[5,10]$. Blood samples of convalescent SARS-CoV-1 infected HCWs were collected from 16 August 2006 to 6 March 2007 (average 3.5 years post-SARS-CoV-1 infection). The serum was separated from blood samples using a centrifuge and stored at $-80{ }^{\circ} \mathrm{C}$ freezer after the time of collection.

\subsection{Neutralization Ability of $n A b s$}

The pseudovirus neutralization assay was conducted according to the protocol published previously [6]. In brief, the SARS-CoV-1 pseudotyped virus normalized for the amount of p24 was incubated with the anti-sera from healthy control group or anti-S or anti-nucleocapsid(N) antibodies from SARS-CoV-1 patients after three years post-infection. After incubation for $1 \mathrm{~h}$, these complexes were added to ACE2-transfected 239T cells. The 
culture was replaced with a fresh medium $24 \mathrm{~h}$ later and incubated for an additional $48 \mathrm{~h}$. Luciferase activity was measured according to the manufacturer's instructions (Promega). The percentage of inhibition assessed by luciferase reporter gene expression was calculated by the reduction in luciferase activity relative to values achieved in the absence of sera. Inhibition seen with control sera was $<10 \%$ in general compared with samples lacking IgG.

\subsection{Statistical Analysis}

Fisher's exact and chi-square tests were performed to determine the statistical significance of factors and anti-SARS-CoV- 1 antibodies' existence status. A value of $p<0.05$ was considered statistically significant. The data analysis was performed using SAS software, Version 9.4 of the SAS System for Windows (SAS Institute, Cary, NC, USA).

\section{Results}

The results indicated that the mean age of participants was 38.7 years (range, 9-81 years). Most of the participants were female $(73.1 \%)$ and did not present acute respiratory distress syndrome (ARDS) (80\%) during SARS-CoV-1 infection (Table 1). To detect the specific antibodies against $\mathrm{S}$ or $\mathrm{N}$ proteins of SARS-CoV-1, immunoblotting was conducted [1]. The results showed that $46.2 \%$ of participants have positive results of anti-N antibody, and $7.7 \%$ of participants showed co-existing anti-S and $\mathrm{N}$ antibodies after 3 years post SARS-CoV-1 infection $(p=0.0003)$. No anti-S antibodies were detected in participants without anti-N antibodies (Table 1).

Table 1. Demographic and clinical characteristics of healthcare workers who were convalescent SARS-CoV-1 patients after three years post-infection in this study.

\begin{tabular}{lccc}
\hline Variable & $\begin{array}{c}\text { Anti-N (+) } \\
\mathbf{N}=\mathbf{6 0} \\
\boldsymbol{n} \mathbf{\%}(\mathbf{)}\end{array}$ & $\begin{array}{c}\text { Anti-N (-) } \\
\mathbf{N}=\mathbf{7 0} \\
\boldsymbol{n} \mathbf{( \% )}\end{array}$ & $\boldsymbol{p}$ \\
\hline Gender & & & $0.3929 \S$ \\
Male & $14(23.3)$ & $21(30.0)$ & \\
Female & $46(76.7)$ & $49(70.0)$ & $0.1546^{*}$ \\
Age & & & \\
$<20$ & $0(0.0)$ & $5(7.1)$ & \\
$20-29$ & $13(21.7)$ & $19(27.1)$ & \\
$30-39$ & $17(28.3)$ & $18(25.7)$ & \\
$40-49$ & $16(26.7)$ & $13(18.6)$ & \\
$50-59$ & $12(20.0)$ & $9(12.9)$ & \\
$\geq 60$ & $2(3.3)$ & $6(8.6)$ & \\
Clinical symptom & & $56(80.0)$ & \\
without ARDS & $48(80.0)$ & $14(20.0)$ & \\
ARDS & $12(20.0)$ & $70(100.0)$ & \\
Anti-S Abs & $50(83.3)$ & $0(0.0)$ & \\
Negative & $10(16.7)$ & & \\
Positive & & & \\
\hline
\end{tabular}

§, Chi-square test; *, Fisher exact test.

We further performed an antibody neutralization assay using SASR-CoV-1 pseudotyped virus to determine whether the existing anti-S antibody had the ability to block SARS-CoV-1 infection. Eight anti-S antibodies from participants showed neutralization ability ( $6.2 \%$ of total, $80 \%$ of anti-S antibody-positive participants). Due to the limited or loss of serums of the anti-N antibody-positive participants, we only tested 24 participants for neutralization ability. The results indicated that all participants with anti-N antibody did not display neutralizing ability (Figure 1). 


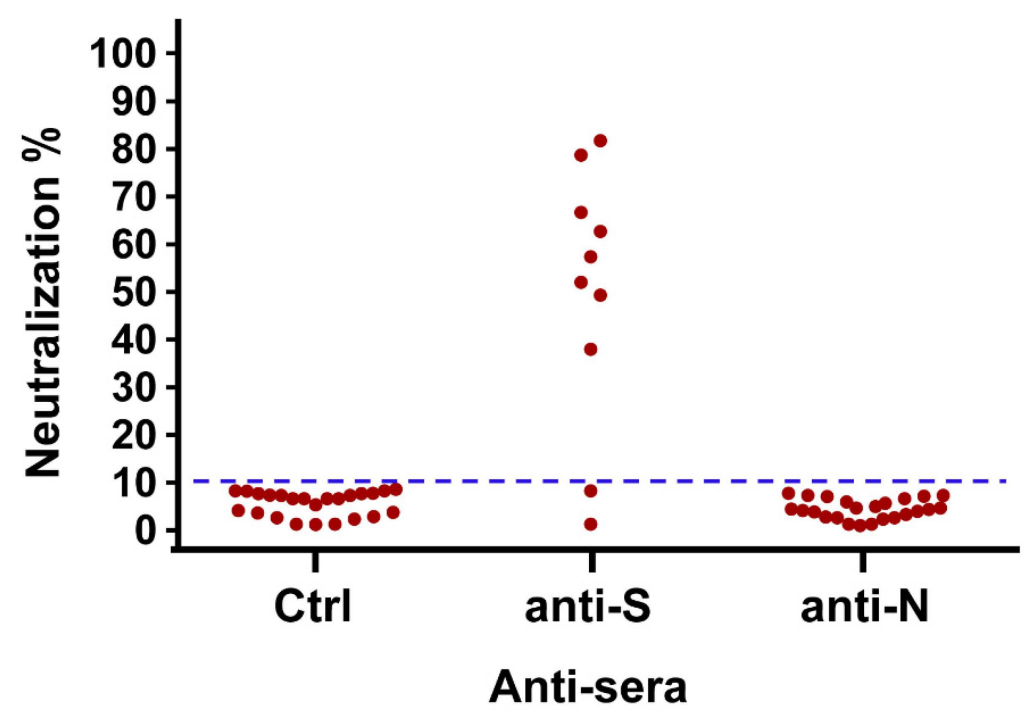

Figure 1. The neutralization ability of anti-sera from convalescent SARS-CoV-1 infected HCWs. The percentage of inhibition assessed by luciferase reporter gene expression was calculated by the reduction in luciferase activity relative to values achieved in the absence of sera. The inhibition seen with control sera was $<10 \%$ in general compared with samples lacking IgG.

Further analysis was done on the factors associated with the existence of anti-SARSCoV-1 antibodies. Results showed that anti-S antibody was significantly present in younger participants $(\leq 49$ years, $p=0.043$ ), whereas there was no anti-S antibody in older participants ( $\geq 50$ years). There are no differences in anti-S antibody existence status with gender or clinical symptom ( $p=1$ and 0.669 , respectively) (Table 2 ). Since the severe disease can provide a higher immune response, we further analyzed the antibodies' existence status in participants with and without ARDS. However, there are no significant differences in anti-S and anti-N antibodies existence in participants with or without ARDS $(p=0.686$ and 1 , respectively; data not shown).

Table 2. Factors associated with neutralizing antibody existence status among the positive result of anti-N antibody participants.

\begin{tabular}{lccc}
\hline Variable & $\begin{array}{c}\text { Anti-S (+) } \\
\mathbf{N = 1 0} \\
\boldsymbol{n} \mathbf{( \% )}\end{array}$ & $\begin{array}{c}\text { Anti-S (-) } \\
\mathbf{N}=\mathbf{5 0} \\
\boldsymbol{n} \mathbf{( \% )}\end{array}$ & $\boldsymbol{p}^{*}$ \\
\hline Gender & & & 1 \\
$\quad$ Male & $2(20.0)$ & $12(24.0)$ & \\
Female & $8(80.0)$ & $38(76.0)$ & 0.0434 \\
Age & & & \\
$\leq 29$ & $1(10.0)$ & $12(24.0)$ & \\
$30-49$ & $9(90.0)$ & $24(48.0)$ & \\
$\geq 50$ & $0(0.0)$ & $14(28.0)$ & \\
Clinical symptom & & $39(78.0)$ & \\
without ARDS & $9(90.0)$ & $11(22.0)$ & \\
$\quad$ ARDS & $1(10.0)$ & & \\
\hline
\end{tabular}

${ }^{*}$ Fisher exact test.

\section{Discussion}

In this retrospective survey, we found that $46.2 \%$ of the participants still had existing anti-N antibodies after three years post SARS-CoV-1 infection. Within the participants with existing anti-N antibodies, there were $16.7 \%$ participants, with co-existing anti-S antibody and $80 \%$ of them have neutralization ability. Most anti-N antibodies did not show neutralization capabilities; however, a higher percentage of these antibodies (46.2\%) were detected in SARS-CoV-1 patients during a long period, suggesting that anti-N antibodies 
may be good candidates for the measurement of SARS-CoV-1 infection during convalescent phase. Although $\mathrm{N}$ protein is not a major target for $\mathrm{nAbs}$ generation, it is a representative antigen for the T-cell response to further induce SARS-specific T-cell proliferation and cytotoxic activity $[11,12]$. The N protein of SARS-CoV-1 and other coronaviruses (such as SARS-CoV-2) are highly conserved and show high immunogenic and abundant expression in infection. The $\mathrm{N}$ protein could be a promising target for vaccine development $[11,12]$.

Previous reports indicated that the nAbs from convalescent SARS-CoV-1 infected patients have been shown to start to drop at month 4 and keep decreasing until month 36 post-infection, and it has been further demonstrated that the kinetics of antibodies do not show a significant difference in clinical characteristics [13]. However, to our knowledge, the current study is the first to demonstrate that age is significantly associated with the SARS-CoV-1 nAbs existence status. A small cohort study that recruited 56 convalescent patients with SARS-CoV-1 showed that all participants presented nAbs up to two years post-infection, but the titer decreased significantly after month 16 . They found nAbs levels significantly decreased in men compared to women at month 24 after SARS-CoV-1 infection, which suggests that gender may be one of the factors associated with the dynamics of antibody responses [14]. Since the $\mathrm{nAbs}$ could significantly wane after two years post-SARSCoV-1 infection [2], the age difference affecting the nAbs existence status could be observed in convalescent SARS-CoV-1 infected patients after three years post-infection based on our results. Other than the $\mathrm{nAbs}$ existence status, the memory B cells and T cells play a vital role against viral re-infection. Several pieces of evidence indicating that the anti-SARS antibodies and peripheral memory B cells responses vanished in convalescent SARS-CoV-1 infected patients after six years post-infection, whereas the SARS-CoV-1 specific memory $T$ cells responses could persist $[3,15,16]$. Therefore, the SARS-CoV- 1 specific memory B cells responses could be a factor affecting the nAbs longevity. The limitations of this study were (1) we do not have multiple points for determining the kinetics of the nAbs and (2) fewer clinical factors and detail medical records of enrolled subjects included in this study. However, there is no significant association linked to the clinical factors and the existence of nAbs after long-term post-SARS-CoV-1 infection reported in other studies.

SARS-CoV-2 pandemic poses great threats for public health and the global economy. Generation of SARS-CoV-2 nAbs from convalescent infected individuals and vaccination is a critical step for prevention of transmission and termination of the pandemic. In convalescent SARS-CoV-2 infected individuals, evidence showed that nAbs can sustain for over a year and exhibited a longer duration of detectable antibody with severe disease patients; however, the nAbs responses were not affected by different age groups [17]. This may be due to individuals infected with SARS-CoV-2 with older age having higher chances to develop severe diseases presentation, thus generating higher titer of nAbs [17-19]. We did not observe statistical differences of anti-N or anti-S existence status in HCWs with and without ARDS in our cohort. Similar observation from another study showed that clinical characteristics did not associate with antibody existence status in long-term SARS-CoV-1 convalescent individuals [13]. Currently, two mRNA-based vaccines (Pfizer and Moderna) and two adenoviral vector-based vaccines (Johnson \& Johnson and Astra-Zeneca) have been widely applied in several countries [20-23]. A recent study by Doria-Rose et. al. showed that the mRNA-1273 vaccine-elicited nAbs can sustain at least six months. Intriguingly, they also found nAbs titers were lower in participants with older age ( $>56$ years old) six months after vaccination [24]. SARS-CoV-2 has evolved to several highly transmissible variants, and one of the most contagious variants, the delta variant (B.1.617.2), has become dominant in circulation and can infect fully vaccinated individuals. Evidence showed that individuals receiving two doses of vaccine can provide similar effectiveness against different SARS-CoV-2 variants (included delta variant); however, only one dose vaccination decreased the effectiveness against delta variant [25]. Therefore, it is believed that a booster dose of COVID-19 is needed to fight against upcoming SARS-CoV-2 variants, especially in vulnerable populations. 
In conclusion, the current study demonstrated that nAbs significantly decreased in convalescent SARS-CoV-1 infected HCWs after three years post-infection, especially with old age. We, therefore, suggested that continuing to monitor nAbs against SARS-CoV-1 or even the current SARS-CoV-2 existence status in infected or immunized persons should be considered, especially in people with older age.

Author Contributions: S.-W.H. and A.N.U. analyzed the data and drafted the manuscript. S.-W.H. and S.-F.W. discussed the concept and designed the manuscript. S.-F.W. and Y.-M.A.C. made critical revisions and gave suggestions of the manuscript. All authors have read and agreed to the published version of the manuscript.

Funding: This work was supported by grants from the Ministry of Science and Technology, R.O.C. (MOST 108-2918-I-037-001, 108-2320-B-037-035-MY3 and MOST 107-2923-B-005-005-MY3) and Kaohsiung Medical University Research Center Grant (KMU-TC109B02).

Institutional Review Board Statement: Ethical clearance for the anonymous use of residual sera samples for the test assessments was provided by the Center for Infectious Disease and Cancer Research, Kaohsiung Medical University. The procedures employed were reviewed and approved by the Ethics Committee of Kaohsiung Medical University (protocol code: KMUHIRB-G(I)20190048, 19 June 2020) without a need for informed consent. The evaluation study was performed in line with the guidelines of the Declaration of Helsinki.

Informed Consent Statement: Not applicable.

Data Availability Statement: All data pertaining to the study was described in the manuscript.

Acknowledgments: The authors wish to thank the staff from the Center for Tropical Medicine and Infectious Disease and Kaohsiung Medical University and professor Yen-Hsu Chen for sample collection, data analysis and IRB application. SWH is supported by a Cancer Research Training Award from the National Cancer Institute (NCI). The content of this publication does not necessarily reflect the views or policies of the NCI, National Institutes of Health, or the Department of Health and Human Services, nor does mention of trade names, commercial products, or organizations imply endorsement by the U.S. Government.

Conflicts of Interest: The authors declare no conflict of interest.

\section{References}

1. Chen, Y.M.; Liang, S.Y.; Shih, Y.P.; Chen, C.Y.; Lee, Y.M.; Chang, L.; Jung, S.Y.; Ho, M.S.; Liang, K.Y.; Chen, H.Y.; et al. Epidemiological and genetic correlates of severe acute respiratory syndrome coronavirus infection in the hospital with the highest nosocomial infection rate in Taiwan in 2003. J. Clin. Microbiol. 2006, 44, 359-365. [CrossRef] [PubMed]

2. Wu, L.P.; Wang, N.C.; Chang, Y.H.; Tian, X.Y.; Na, D.Y.; Zhang, L.Y.; Zheng, L.; Lan, T.; Wang, L.F.; Liang, G.D. Duration of antibody responses after severe acute respiratory syndrome. Emerg. Infect. Dis. 2007, 13, 1562-1564. [CrossRef]

3. Tang, F.; Quan, Y.; Xin, Z.T.; Wrammert, J.; Ma, M.J.; Lv, H.; Wang, T.B.; Yang, H.; Richardus, J.H.; Liu, W.; et al. Lack of peripheral memory B cell responses in recovered patients with severe acute respiratory syndrome: A six-year follow-up study. J. Immunol. 2011, 186, 7264-7268. [CrossRef] [PubMed]

4. Martínez-Flores, D.; Zepeda-Cervantes, J.; Cruz-Reséndiz, A.; Aguirre-Sampieri, S.; Sampieri, A.; Vaca, L. SARS-CoV-2 Vaccines Based on the Spike Glycoprotein and Implications of New Viral Variants. Front. Immunol. 2021, 12. [CrossRef] [PubMed]

5. Wang, S.F.; Chen, K.H.; Chen, M.; Li, W.Y.; Chen, Y.J.; Tsao, C.H.; Yen, M.Y.; Huang, J.C.; Chen, Y.M. Human-leukocyte antigen class I CW 1502 and class II DR 0301 genotypes are associated with resistance to severe acute respiratory syndrome (SARS) infection. Viral Immunol. 2011, 24, 421-426. [CrossRef]

6. Lu, R.; Zhao, X.; Li, J.; Niu, P.; Yang, B.; Wu, H.; Wang, W.; Song, H.; Huang, B.; Zhu, N.; et al. Genomic characterisation and epidemiology of 2019 novel coronavirus: Implications for virus origins and receptor binding. Lancet 2020, 395, 565-574. [CrossRef]

7. Hoffmann, M.; Kleine-Weber, H.; Schroeder, S.; Kruger, N.; Herrler, T.; Erichsen, S.; Schiergens, T.S.; Herrler, G.; Wu, N.H.; Nitsche, A.; et al. SARS-CoV-2 Cell Entry Depends on ACE2 and TMPRSS2 and Is Blocked by a Clinically Proven Protease Inhibitor. Cell 2020, 181, 271-280.e278. [CrossRef] [PubMed]

8. Tian, X.; Li, C.; Huang, A.; Xia, S.; Lu, S.; Shi, Z.; Lu, L.; Jiang, S.; Yang, Z.; Wu, Y.; et al. Potent binding of 2019 novel coronavirus spike protein by a SARS coronavirus-specific human monoclonal antibody. Emerg. Microbes Infect. 2020, 9, 382-385. [CrossRef]

9. Tai, W.; Zhang, X.; He, Y.; Jiang, S.; Du, L. Identification of SARS-CoV RBD-targeting monoclonal antibodies with cross-reactive or neutralizing activity against SARS-CoV-2. Antivir. Res. 2020, 179, 104820. [CrossRef]

10. Yang, Z.Y.; Werner, H.C.; Kong, W.P.; Leung, K.; Traggiai, E.; Lanzavecchia, A.; Nabel, G.J. Evasion of antibody neutralization in emerging severe acute respiratory syndrome coronaviruses. Proc. Natl. Acad. Sci. USA 2005, 102, 797-801. [CrossRef] 
11. Dutta, N.K.; Mazumdar, K.; Gordy, J.T. The Nucleocapsid Protein of SARS-CoV-2: A Target for Vaccine Development. J. Virol. 2020, 94, e00647-20. [CrossRef]

12. Gao, W.; Tamin, A.; Soloff, A.; D'Aiuto, L.; Nwanegbo, E.; Robbins, P.D.; Bellini, W.J.; Barratt-Boyes, S.; Gambotto, A. Effects of a SARS-associated coronavirus vaccine in monkeys. Lancet 2003, 362, 1895-1896. [CrossRef]

13. Cao, W.C.; Liu, W.; Zhang, P.H.; Zhang, F.; Richardus, J.H. Disappearance of antibodies to SARS-associated coronavirus after recovery. N. Engl. J. Med. 2007, 357, 1162-1163. [CrossRef] [PubMed]

14. Liu, W.; Fontanet, A.; Zhang, P.H.; Zhan, L.; Xin, Z.T.; Baril, L.; Tang, F.; Lv, H.; Cao, W.C. Two-year prospective study of the humoral immune response of patients with severe acute respiratory syndrome. J. Infect. Dis. 2006, 193, 792-795. [CrossRef]

15. Fan, Y.Y.; Huang, Z.T.; Li, L.; Wu, M.H.; Yu, T.; Koup, R.A.; Bailer, R.T.; Wu, C.Y. Characterization of SARS-CoV-specific memory T cells from recovered individuals 4 years after infection. Arch. Virol. 2009, 154, 1093-1099. [CrossRef] [PubMed]

16. Peng, H.; Yang, L.T.; Wang, L.Y.; Li, J.; Huang, J.; Lu, Z.Q.; Koup, R.A.; Bailer, R.T.; Wu, C.Y. Long-lived memory T lymphocyte responses against SARS coronavirus nucleocapsid protein in SARS-recovered patients. Virology 2006, 351, 466-475. [CrossRef]

17. Lau, E.H.Y.; Tsang, O.T.Y.; Hui, D.S.C.; Kwan, M.Y.W.; Chan, W.H.; Chiu, S.S.; Ko, R.L.W.; Chan, K.H.; Cheng, S.M.S.; Perera, R.; et al. Neutralizing antibody titres in SARS-CoV-2 infections. Nat. Commun. 2021, 12, 63. [CrossRef]

18. Xiang, T.; Liang, B.; Fang, Y.; Lu, S.; Li, S.; Wang, H.; Li, H.; Yang, X.; Shen, S.; Zhu, B.; et al. Declining Levels of Neutralizing Antibodies Against SARS-CoV-2 in Convalescent COVID-19 Patients One Year Post Symptom Onset. Front Immunol. 2021, 12, 708523. [CrossRef]

19. Chia, W.N.; Zhu, F.; Ong, S.W.X.; Young, B.E.; Fong, S.W.; Le Bert, N.; Tan, C.W.; Tiu, C.; Zhang, J.; Tan, S.Y.; et al. Dynamics of SARS-CoV-2 neutralising antibody responses and duration of immunity: A longitudinal study. Lancet. Microbe 2021, 2, e240-e249. [CrossRef]

20. Polack, F.P.; Thomas, S.J.; Kitchin, N.; Absalon, J.; Gurtman, A.; Lockhart, S.; Perez, J.L.; Perez Marc, G.; Moreira, E.D.; Zerbini, C.; et al. Safety and Efficacy of the BNT162b2 mRNA Covid-19 Vaccine. N. Engl. J. Med. 2020, 383, 2603-2615. [CrossRef]

21. Baden, L.R.; El Sahly, H.M.; Essink, B.; Kotloff, K.; Frey, S.; Novak, R.; Diemert, D.; Spector, S.A.; Rouphael, N.; Creech, C.B.; et al. Efficacy and Safety of the mRNA-1273 SARS-CoV-2 Vaccine. N. Engl. J. Med. 2021, 384, 403-416. [CrossRef] [PubMed]

22. Voysey, M.; Costa Clemens, S.A.; Madhi, S.A.; Weckx, L.Y.; Folegatti, P.M.; Aley, P.K.; Angus, B.; Baillie, V.L.; Barnabas, S.L.; Bhorat, Q.E.; et al. Single-dose administration and the influence of the timing of the booster dose on immunogenicity and efficacy of ChAdOx1 nCoV-19 (AZD1222) vaccine: A pooled analysis of four randomised trials. Lancet 2021, 397, 881-891. [CrossRef]

23. Sadoff, J.; Gray, G.; Vandebosch, A.; Cardenas, V.; Shukarev, G.; Grinsztejn, B.; Goepfert, P.A.; Truyers, C.; Fennema, H.; Spiessens, B.; et al . Safety and Efficacy of Single-Dose Ad26.COV2.S Vaccine against Covid-19. N. Engl. J. Med. 2021, 384, 2187-2201. [CrossRef] [PubMed]

24. Doria-Rose, N.; Suthar, M.S.; Makowski, M.; O'Connell, S.; McDermott, A.B.; Flach, B.; Ledgerwood, J.E.; Mascola, J.R.; Graham, B.S.; Lin, B.C.; et al. Antibody Persistence through 6 Months after the Second Dose of mRNA-1273 Vaccine for Covid-19. N. Engl. J. Med. 2021, 384, 2259-2261. [CrossRef]

25. Lopez Bernal, J.; Andrews, N.; Gower, C.; Gallagher, E.; Simmons, R.; Thelwall, S.; Stowe, J.; Tessier, E.; Groves, N.; Dabrera, G.; et al. Effectiveness of Covid-19 Vaccines against the B.1.617.2 (Delta) Variant. N. Engl. J. Med. 2021. [CrossRef] 